\title{
POLYNOMIAL HARMONIC MORPHISMS BETWEEN EUCLIDEAN SPHERES
}

\author{
JAMES EELLS AND PAUL YIU
}

(Communicated by Peter Li)

\begin{abstract}
A characterization is given of the harmonic morphisms between euclidean spheres whose component functions are harmonic homogeneous polynomials of the same degree, and also of polynomial harmonic morphisms between euclidean spaces which map spheres into spheres. These turn out to be isometric to the classical Hopf fibrations.
\end{abstract}

\section{INTRODUCTION}

A harmonic morphism is a map $\phi:(M, g) \rightarrow(N, h)$ between riemannian manifolds that preserves germs of harmonic functions: for each function $f$ which is harmonic on an open set $V \subseteq N$, the composite $f \circ \phi$ is a harmonic function on $\phi^{-1}(V)$. See, for example, [EL1, (4.12)]. Fuglede [F] and Ishihara [I] have shown that $\phi:(M, g) \rightarrow(N, h)$ is a harmonic morphism if and only if it is harmonic and horizontally conformal. This latter property means that on the horizontal part of each tangent space, i.e., the orthogonal complement of $\operatorname{ker}\left(d \phi_{x}\right) \subseteq T_{x} M$, the differential restricts to a similarity. In other words, there is a dilation function $\mu: M \rightarrow \mathbb{R}^{+}$such that

$$
\left\|d \phi_{x}(u)\right\|_{h}=\mu(x)\|u\|_{g} \quad \text { for every } u \in\left(\operatorname{ker} d \phi_{x}\right)^{\perp} .
$$

In this note, we prove the following theorems.

Theorem 1. Let $m>n$, and let $\phi: S^{m} \rightarrow S^{n}$ be a map whose component functions are all harmonic homogeneous polynomials of the same degree $k$. The map $\phi$ is a harmonic morphism if and only if it is isometric to one of the classical Hopf fibrations $S^{3} \rightarrow S^{2}, S^{7} \rightarrow S^{4}$, or $S^{15} \rightarrow S^{8}$.

Theorem 2. Let $m>n$, and let $\phi: S^{m} \rightarrow S^{n}$ be the restriction of a homogeneous polynomial harmonic morphism $\Phi: \mathbb{R}^{m+1} \rightarrow \mathbb{R}^{n+1}$. Then $\phi$ is isometric to one of the classical Hopf fibrations.

Received by the editors February 14, 1994.

1991 Mathematics Subject Classification. Primary 58E20; Secondary 55R25.

Key words and phrases. Harmonic morphisms, Hopf fibrations, orthogonal multiplications, polynomial maps between spheres.

The second author was partially supported by NSF Grant DMS-9201204.

(c) 1995 American Mathematical Society 


\section{ORTHOGONAL MULTIPLICATIONS}

Baird [B1, Theorem 7.2.7] has shown that an orthogonal multiplication $f$ : $\mathbb{R}^{r} \times \mathbb{R}^{s} \rightarrow \mathbb{R}^{n}$, i.e., a bilinear map satisfying

$$
\|f(x, y)\|=\|x\|\|y\|, \quad x \in \mathbb{R}^{r}, y \in \mathbb{R}^{s},
$$

is a harmonic morphism if and only if $r=s=n=1,2,4$ or 8. Given an orthogonal multiplication $f$ as in (1), there is a homogeneous quadratic map $F: S^{r+s-1} \rightarrow S^{n}$, called the Hopf construction of $f$, defined by

(3) $F(x, y)=\left(\|x\|^{2}-\|y\|^{2}, 2 f(x, y)\right), \quad x \in \mathbb{R}^{r}, y \in \mathbb{R}^{s},\|x\|^{2}+\|y\|^{2}=1$.

If $r=s$, then $F: S^{2 r-1} \rightarrow S^{n}$ is always a harmonic map. Gigante [EL3, (10.5)] has shown that $F$ is a harmonic morphism if and only if $r=s=n=1,2,4$ or 8 .

The standard multiplications in the real division algebras $\mathbb{R}, \mathbb{C}, \mathbb{H}$ and (1) give orthogonal multiplications $f: \mathbb{R}^{n} \times \mathbb{R}^{n} \rightarrow \mathbb{R}^{n}$ for $n=1,2,4,8$. Hopf's construction on these $f$ yields the classical Hopf fibrations $S^{2 n-1} \rightarrow S^{n}$ referred to in the statements of Theorems 1 and 2.

There are many orthogonal multiplications in those dimensions. Indeed, from $[\mathrm{KR}]^{1}$

(a) those $f$ with 2-sided unit are isometrically isomorphic to the multiplication in $\mathbb{R}, \mathbb{C}, \mathbb{H}$ and $\mathbb{O}$ according to a classical theorem of Hurwitz;

(b) with any orthogonal multiplication $f: \mathbb{R}^{n} \times \mathbb{R}^{n} \rightarrow \mathbb{R}^{n}$ and any $a \in S^{n-1}$ we can associate an orthogonal multiplication $f_{a}$ with a 2-sided unit, via the following construction:

Define the isometries

$$
\lambda(x)=f(f(a, a), x), \quad \rho(x)=f(x, f(a, a)),
$$

and let

$$
f_{a}(x, y)=f\left(\rho^{-1}(x), \lambda^{-1}(y)\right), \quad e=f(f(a, a), f(a, a)) .
$$

Then, $\rho^{-1}(e)=f(a, a)=\lambda^{-1}(e)$, and routine calculations reveal that $f_{a}$ : $\mathbb{R}^{n} \times \mathbb{R}^{n} \rightarrow \mathbb{R}^{n}$ is an orthogonal multiplication with 2-sided unit $e$.

In conjunction with the following proposition, that solves Problem (4.7) of [EL2].

Proposition 3. A homogeneous quadratic map $\phi: S^{m} \rightarrow S^{n}$ is a harmonic morphism if and only if it is isometric to one of the classical Hopf fibrations $S^{2 n-1} \rightarrow S^{n}$ for $n=1,2,4,8$.

That was established by the second author [Y, Remark following Proposition 4.3]; its proof is elaborated in that of Theorem 1 below.

\section{Proof of Theorem 1}

The classical Hopf fibrations are all harmonic morphisms: each of them is harmonic and horizontally conformal with a constant dilation function $\mu(x)=$ 2 satisfying (1). Conversely, let $\phi: S^{m} \rightarrow S^{n}$ be a harmonic morphism with component functions harmonic homogeneous polynomials of degree $k$. These restrict to eigenfunctions of the Laplacian $\Delta^{S^{m}}$ with eigenvalue $k(k+m-1)$

\footnotetext{
${ }^{1}$ The first author is indebted to Dr. A. Sudbery for the reference [KR], and for related instruction.
} 
([ER, Corollary VIII.1.4]). It follows that $\phi$ is indeed an eigenmap with constant energy density function

$$
e(\phi)=\frac{1}{2}\|d \phi\|^{2}=\frac{1}{2} k(k+m-1) .
$$

Note that $\left\|d \phi_{x}\right\|^{2}=\sum_{u}\left\|d \phi_{x}(u)\right\|^{2}$ with summation over an orthonormal basis of $T_{x}\left(S^{m}\right)$. Let $\mu: S^{m} \rightarrow \mathbb{R}^{+}$denote the dilation function of the harmonic morphism $\phi$. From (4) above we infer that $\phi$ cannot have vanishing differential at any point of $S^{m}$. In other words, the dilation function $\mu$ is nowhere zero. Thus, for each $x \in S^{m}$, the differential $d \phi_{x}$ has full rank $n$, and satisfies

$$
\left\|d \phi_{x}\right\|^{2}=n \cdot \mu(x)^{2} \text {. }
$$

It follows that the dilation function $\mu$ is constant:

$$
\mu(x)=\sqrt{\frac{k(k+m-1)}{n}}
$$

Since a harmonic morphism is an open mapping, $\phi: S^{m} \rightarrow S^{n}$ must be surjective. Also, since $d \phi_{x}$ are all surjective, $\phi$ is a submersion, and therefore a locally trivial fibration. By Browder's theorem [Br],

$$
(m, n)=(3,2),(7,4),(15,8),
$$

the dimensions of the classical Hopf fibrations. Indeed, Hsu $[\mathrm{H}]$ has shown that each $\phi^{-1}(x)$ is a compact, connected manifold in $S^{m}$. Following Hsu, by tracing a horizontal geodesic joining a point $x \in S^{m}$ to its antipode $-x$, we see that $\mu$ is a positive even integer. For $r>0$, denote by $B_{x}(r)$ the open $n$-ball consisting of all points on $S^{m}$ at a distance $<r$ from $x$, traced out along horizontal tangential directions. Note that $B_{x}\left(\frac{\pi}{\mu}\right)$ is mapped diffeomorphically onto $S^{n}-\{-x\}$. Note also that for each $l=0,1, \ldots,\left[\frac{\mu-1}{2}\right], \phi$ sends the $(n-1)$-sphere $\partial B_{x}\left(\frac{(2 l+1) \pi}{\mu}\right)$ to the point $-\phi(x)$. Since each of these spheres is both open and closed (the latter by the invariance of domain) in the compact connected manifold $M=\phi^{-1}(-\phi(x))$, there can be only one of them. From this, we conclude that $\mu=2$, and $k=2$ from (5). The components of the map $\phi$ are homogeneous quadratic polynomials.

In [Y], it was shown that if $\phi: S^{m} \rightarrow S^{n}$ is a homogeneous quadratic map, then, for every point $q \in S^{n}$ in the image of $\phi$, the inverse image $\phi^{-1}(q)$ is a great sphere cut out by a linear subspace $W_{q} \subset \mathbb{R}^{m+1}$, the dimension of $W_{q}$ being $m+1-$ rank $d \phi_{v}$ for any $v \in \phi^{-1}(q)$.

By Proposition 3.2 of [Y], the homogeneous quadratic map $\phi$ is the Hopf construction of some orthogonal multiplication $f: \mathbb{R}^{r} \times \mathbb{R}^{s} \rightarrow \mathbb{R}^{n}$ if and only if there exists a pair of antipodal points $\pm q \in S^{n}$ such that

$$
\operatorname{dim} W_{q}+\operatorname{dim} W_{-q}=m+1 \text {. }
$$

Here, $r=\operatorname{dim} W_{q}$ and $s=\operatorname{dim} W_{-q}$. In the present case, this is clearly satisfied since each pair of antipodal points $\pm q \in S^{n}$ satisfies

$$
\operatorname{dim} W_{q}=\operatorname{dim} W_{-q}=m-n+1=\frac{1}{2}(m+1)
$$


for $(m, n)$ given in (6). We conclude, therefore, that the harmonic morphism $\phi: S^{2 n-1} \rightarrow S^{n}$ is indeed the Hopf construction of an orthogonal multiplication $\mathbb{R}^{n} \times \mathbb{R}^{n} \rightarrow \mathbb{R}^{n}$. As such, it is isometric to the classical Hopf fibration.

\section{Proof of Theorem 2}

Baird ([B1, §8.1], with correction in [B2, §4]) has shown that if $\Phi: \mathbb{R}^{m+1} \rightarrow$ $\mathbb{R}^{n+1}$ is a nonconstant $k$-homogeneous polynomial harmonic morphism, then

$$
m-1 \geq k(n-1) \text {. }
$$

Suppose that $\Phi$ restricts to a map $\phi: S^{m} \rightarrow S^{n}$; then by a theorem of R. Wood ([W, p.163]),

$$
m<2 n \text {. }
$$

Combining (7) and (8), we obtain $2 n-1>m-1 \geq k(n-1)$. Therefore,

$$
k=2 \text { and } m=2 n-1 \text {. }
$$

The same reasoning as in the proof of Theorem 1 completes the proof.

\section{REMARKS ON GENERAL HARMONIC MORPHISMS BETWEEN SPHERES}

(1) Let $\eta: S^{3} \rightarrow S^{2}$ be the standard Hopf fibration and $\theta_{r}: S^{2} \rightarrow S^{2}$ a holomorphic map of degree $r \geq 2$. Then, $\eta_{r}=\theta_{r} \circ \eta: S^{3} \rightarrow S^{2}$ is a harmonic morphism with critical points. It is certainly not a Hopf fibration. However, the classification theorem of [BW] shows that all harmonic morphisms $\phi: S^{3} \rightarrow S^{2}$ are of the form $\phi=\theta \circ \eta$ for a suitable holomorphic (anti-holomorphic) map.

(2) In the context of topology, if $\phi: S^{m} \rightarrow S^{n}$ is a locally trivial fibration with $m>n \geq 2$, then $m=2 n-1$ and $n=2,4,8$. The fibre model $F$ has the homotopy type of $S^{n-1}$. For $n=4,8$, there are many such smooth fibrations [EK]. We do not know how to identify the harmonic morphisms amongst them.

(3) A proper submersion is a locally trivial fibration, and so occurs only in the dimensions $(m, n)=(3,2),(7,4),(15,8)$ of the Hopf fibrations. However, Baird [B2] has shown that if $\phi:\left(M^{m}, g\right) \rightarrow\left(N^{n}, h\right)$ is a nonconstant harmonic morphism, and if $m<2(n-1)$, then $\phi$ is a submersion. Thus, we obtain

Proposition 4. If $n<m<2 n-2$, any harmonic morphism $\phi: S^{m} \rightarrow S^{n}$ is constant.

The special case $m=n+1$ and $n>3$ is due to [B2]. We observe that the proof of the proposition is valid for exotic spheres, and for arbitrary riemannian metrics. Finally, we remark that Baird [B2, §5] and Baird and Ratto [BR] have constructed nonconstant harmonic morphisms on deformed spheres in dimensions $(m, n)=(4,3),(8,5)$ and $(16,9)$.

\section{REFERENCES}

[B1] P. Baird, Harmonic maps with symmetry, harmonic morphisms and deformations of metrics, Res. Notes in Math., vol. 87, Pitman, London, 1983.

[B2] Harmonic morphisms and circle actions on 3 - and 4 -manifolds, Ann. Inst. Fourier (Grenoble) 40 (1990), 177-212.

[BR] P. Baird and A. Ratto, Conservation laws, equivariant harmonic maps and harmonic morphisms, Proc. London Math. Soc. (3) 64 (1992), 197-224. 
[BW] P. Baird and J.C. Wood, Bernstein theorems for harmonic morphisms from $\mathbb{R}$ and $S^{3}$, Math. Ann. 280 (1988), 579-603.

[Br] W. Browder, Higher torsion in H-spaces, Trans. Amer. Math. Soc. 108 (1965), 353-375.

[EK] J. Eells and N. Kuiper, An invariant for certain smooth manifolds, Ann. Mat. Pura Appl. 60 (1963), 93-110.

[EL1] J. Eells and L. Lemaire, A report on harmonic maps, Bull. London Math. Soc. 10 (1978), $1-68$.

[EL2] __ Topics in harmonic maps, CBMS Regional Conf. Ser. in Math., vol. 50, Amer. Math. Soc., Providence, RI, 1983.

[EL3] _ Another report on harmonic maps, Bull. London Math. Soc. 20 (1988), 385-524.

[ER] J. Eells and A. Ratto, Harmonic maps and minimal immersions with symmetries, Ann. of Math. Studies, vol. 130, Princeton Univ. Press, Princeton, NJ, 1993.

[F] B. Fuglede, Harmonic morphisms between Riemannian manifolds, Ann. Inst. Fourier (Grenoble) 28 (1978), 107-144.

[G] G. Gigante, A note on harmonic morphisms, preprint, 1983.

[H] A.C.L. Hsu, A characterization of the Hopf map by stretch, Math. Z. 129 (1972), 195-206.

[I] T. Ishihara, A mapping of Riemannian manifolds which preserves harmonic functions, J. Math. Kyoto Univ. 19 (1979), 215-229.

[KR] M. Koecher and R. Remmert, "Composition algebras. Hurwitz's theorem-vector product algebras", Chapter 10 in H.-D. Ebbinghaus et al., Numbers, Graduate Texts in Math., vol. 123, Springer-Verlag, Berlin, 1990, pp. 265-280.

[W] R. Wood, Polynomial maps from spheres to spheres, Invent. Math. 5 (1968), 163-168.

[Y] P. Yiu, Quadratic forms between spheres and the non-existence of sums of squares formulae, Math. Proc. Cambridge Philos. Soc. 100 (1986), 493-504.

10 Redwood Lodge, Grange Road, Cambridge CB3 9AR, England

Department of Mathematics, Florida Atlantic University, Boca Raton, Florida 33431, USA

E-mail address: yiu@acc.fau.edu 